\title{
Computational Fluid Dynamics Simulations of a GO2/GH2 Single Element Combustor
}

\author{
Victor P. Zhukov* \\ German Aerospace Center (DLR), 74239 Hardthausen, Germany
}

\begin{abstract}
DOI: $10.2514 / 1 . B 35654$
A single-element combustor known as the "Penn State preburner combustor" is modeled numerically using the commercial computational fluid dynamics code ANSYS CFX. The aim of computational fluid dynamics modeling is to simulate the wall heat flux, which has been measured experimentally. The simulated combustion chamber has a single shear coaxial injector and operates with gaseous oxygen and hydrogen in a staged combustion configuration. The turbulent flow in the combustion chamber is modeled using the Favre-averaged Navier-Stokes equations and the shear-stress transport turbulence model. The turbulent non-premixed flame is modeled using an extended eddy dissipation model. The developed turbulent combustion model shows good agreement with the experimental data, good convergence, and a short computational time. A mesh convergence study is performed, and a mesh-independent solution is obtained on a mesh with $\mathbf{1 . 5}$ million nodes. The complexity of the model is gradually increased until the model is capable of predicting the wall heat flux. The analysis of numerical results shows a significant effect of boundary conditions on wall heat flux predictions. The comparison of the Reynolds-averaged Navier-Stokes simulations with the experimental data demonstrates the capability of Reynolds-averaged Navier-Stokes simulations to predict wall heat fluxes in a rocket combustion chamber.
\end{abstract}

\section{Nomenclature \\ $y^{+}=$nondimensional wall distance}

\section{Introduction}

A CCURATE prediction of heat loads is one of the key problems of rocket engine design. At the current moment, the design of rocket combustion chambers requires an extensive trial-and-error testing. Computational fluid dynamics (CFD) modeling offers a means to reduce the amount of expensive hot-fire tests. Once validated, the numerical model can be applied to many other cases. The scope of the present work is the accurate prediction of the wall heat fluxes in a rocket combustion chamber. The goal of the work is to validate our CFD model against the Penn State test case (also known as RCM-1) [1]. This test case is designed for the validation of wall heat flux predictions in a rocket engine combustion chamber.

The Penn State test case is a widely cited experiment carried out at the Pennsylvania State University [1]. In the experiment, wall heat flux measurements were performed in a cylindrical rocket chamber with a single coaxial injection element. The experimental setup consisted of two preburners and a main combustion chamber. The configuration of the experimental setup corresponded to a staged combustion cycle operating with gaseous oxygen and hydrogen propellants. The wall heat flux measurements were recorded using arrays of Gardon-type heat flux gauges and coaxial thermocouples. Since the wall heat flux measurements were the only goal of the experiment, other diagnostics were not used. Drawings and photographs of the test rig along with details of the experiment can be found in [1-3] .

Tucker et al. [3] initiated a cooperative study between five scientific centers. The aim of the study was to compare different CFD models on a standard test case in predicting wall heat flux, contrasting several methods with each other and with experimental data [1]. The simulations conducted for the study consisted of a Reynoldsaveraged Navier-Stokes (RANS) simulation, an unsteady Reynolds-

Received 6 November 2014; revision received 23 April 2015; accepted for publication 1 May 2015; published online 23 July 2015. Copyright $\odot 2015$ by V. P. Zhukov. Published by the American Institute of Aeronautics and Astronautics, Inc., with permission. Copies of this paper may be made for personal or internal use, on condition that the copier pay the $\$ 10.00$ per-copy fee to the Copyright Clearance Center, Inc., 222 Rosewood Drive, Danvers, MA 01923; include the code 1533-3876/15 and \$10.00 in correspondence with the CCC.

*Research Associate, Institute of Space Propulsion; victor.zhukov@ dlr.de. averaged Navier-Stokes (URANS) simulation, and three different large-eddy simulations (LESs). Different meshes with sizes between 250,000 and 255 million cells were used. The chemical reactions of propellants were modeled with the use of detailed chemical kinetics. The RANS simulation was the simplest among the performed simulations. The best agreement with the experimental data was achieved in LES at Sandia National Laboratories. This simulation was performed on a mesh of 255 million cells and required around 2 million CPU hours. The RANS simulation performed at NASA Marshall Space Flight Center required only 1600 CPU hours; nevertheless, the results were in acceptable agreement with experimental data [1]. Unfortunately, the study did not give the answer on why the agreement with the experimental data was not reached in the other three simulations.

Later, Sozer et al. [4] carried out a study aimed at assessing the capability of RANS simulations for CFD modeling of rocket combustion chambers with single coaxial injectors. One of the two test cases chosen for the study was Penn State test case [1]. The CFD simulations were performed using the shear-stress-transport (SST) turbulence model in a RANS framework. Different finite-rate chemical kinetic models were used. Sozer et al. [4] studied the impact of the numerical grid, boundary conditions, wall treatment, and reaction kinetic mechanisms on numerical results. In the Penn State test case, Sozer et al. obtained comparable results to the RANS results in the work of Tucker et al. [3] . It was also shown that, for the Penn State test case, the numerical results were sensitive to mesh resolution in the range of mesh size from 100,000 to 500,000 cells.

Ivancic et al. [5] carried out a cooperative work between two research groups in Germany: Airbus Defence and Space (Airbus D\&S) and the Institute of Aerodynamics and Flow Technology of the German Aerospace Center (DLR-AS). They performed CFD simulations of the Penn State combustor using RANS modeling and employing three different CFD codes: CFX (commercial CFD code of ANSYS) [6,7], Rocflam3 (in-house CFD code under development by Airbus D\&S , and TAU code [in-house CFD code of the German Aerospace Center (DLR)] [8]. Ivancic et al. [5] achieved a good agreement with the experimental data for each of the applied CFD codes; thereby, they showed that the Penn State test case [1] could be modeled using RANS in a two-dimensional domain. To perform CFD simulations of rocket combustion chambers, Airbus D\&S expanded CFX with real gas data, sophisticated mixing rules for viscosity and heat conductivity, and the equilibrium-based combustion model. In the case of CFX and Rocflam3, the agreement with the experiment was achieved by the optimization of the turbulent Prandtl and Schmidt numbers. As a result of such an approach, in 
Table 1 Values of the turbulent Prandtl and Schmidt numbers, which were used in the work of Ivancic et al. [5]

\begin{tabular}{lcc}
\hline \hline Code & $P r_{t}$ & $S c_{t}$ \\
\hline CFX, Airbus D\&S & 0.85 & 0.85 \\
Rocflam3, Airbus D\&S & 0.9 & 0.6 \\
TAU, DLR-AS & $0.78-0.85$ & 0.7 \\
CFX, this work & $0.9^{\mathrm{a}}$ & $0.9^{\mathrm{a}}$ and $0.7^{\mathrm{b}}$ \\
\hline \hline
\end{tabular}

${ }^{\mathrm{a}}$ Value by default in CFX.

${ }^{\mathrm{b}}$ Used in the final simulations.

each code, the different values of the turbulent Prandtl and Schmidt numbers were used; see Table 1 . (The turbulent Prandtl and Schmidt numbers are responsible for the turbulent heat and mass transfer, respectively.) In the case of TAU, the agreement was reached by introducing Durbin's limiter [9] in order to bound the diagonal components of the Reynolds stress tensor. The simulations were performed using different numerical meshes, different types of RANS solvers, different boundary conditions, etc. Under these conditions, it is difficult to draw conclusions from the paper of Ivancic et al. [5]. However, they have clearly demonstrated the significant influence of the turbulent Prandtl and Schmidt numbers, as well as the flow recirculation in the chamber on the results of the simulations.

Ivancic et al. [5] found that the inlet boundary conditions provided by Pal et al. [1] are inconsistent with the measured chamber pressure (the inlet boundary conditions can be found in Table 2). Ivancic et al. [5] showed that the measured chamber pressure of 54.2 bar corresponds to a combustion efficiency of $112.5 \%$. This inconsistency of the inlet boundary conditions with the measured chamber pressure means that the pressure predicted with the use of the given boundary conditions should be lower in CFD simulations than in the experiment. Ivancic et al. [5] assumed that the contradiction between the inlet boundary conditions and the measured chamber pressure arose due to incomplete combustion in the preburners. The inconsistency of the inlet boundary conditions may also have an impact on the wall heat flux, which is the main target of the simulations. The wall heat flux in the Penn State combustor correlates with pressure as $q_{w} \propto p^{0.8}$ [2] (i.e., lower pressure means lower wall heat flux). Thus, the boundary conditions may lead to the lower wall heat flux in the simulations.

In previous work, the development of a CFD model was started for wall heat flux predictions [10]. In this work, the flow in a rocket combustion chamber with a porous injector head is simulated, which is a concept under development at the Institute of Space Propulsion of the German Aerospace Center in Lampoldshausen (DLR-RA). In the porous injector head, hydrogen is injected through a porous plate while oxygen is fed through many small tubes. It was found that wall heat fluxes are very sensitive to the arrangement of the injectors (in this case, oxygen injectors) in an outer row; thus, accurate wall heat flux predictions require a three-dimensional modeling [10]. In other words, wall heat flux can be predicted accurately in two-dimensional (2-D) simulations only if the injector head is axisymmetric. Threedimensional (3-D) simulations require a numerical mesh with numerous nodes and, consequently, more computational power. In

Table 2 Inlet boundary conditions [1]

\begin{tabular}{lcc}
\hline \hline & Oxidizer inlet & Fuel inlet \\
\hline Mass flow rate, $\mathrm{g}$ /s & Total: 90.4 & Total: 33.1 \\
& $\mathrm{O}_{2}: 85.5$ & $\mathrm{H}_{2}: 13.3$ \\
& $\mathrm{H}_{2} \mathrm{O}: 4.98$ & $\mathrm{H}_{2} \mathrm{O}: 19.8$ \\
Temperature, $\mathrm{K}$ & 700 & 811 \\
Pressure, bar & 58.5 & 65.0 \\
Mass ratio of oxidizer to fuel (ROF) & 161 & 1.13 \\
Pressure in main combustor, bar & \multicolumn{2}{c}{54.2} \\
Overall ROF & \multicolumn{2}{c}{6.6} \\
\hline \hline
\end{tabular}

${ }^{a}$ Used in the present simulations as boundary conditions. such a situation, the computational efficiencies of the numerical model and solver are important. To simulate the flow in the combustion chamber with the porous injector plate, we used an eddydissipation-type combustion model [11], which demonstrated a high computational efficiency in comparison with other combustion models (e.g., finite-rate chemistry model, turbulent flame closure model, etc.). The computations on a mesh of 1.4 million nodes only required one night on a single workstation: $5 \mathrm{~h}$ on the Intel Xeon E5645 processor. The aim of the current work is the further development and validation of this combustion model along with other parts of the current CFD model. The Penn State test case has been chosen for the present simulations because it gives an opportunity to compare the approach to model rocket combustion chambers with methods of other research groups and to assess the model in terms of accuracy and computational cost.

\section{Modeling and Numerical Setup}

The models and numerical setup, which are used in the present work, are built upon the model and numerical setup used in earlier work [10]. The employed models and numerical setup are based on the capabilities provided by commercial CFD code ANSYS CFX using user-defined expressions and functions [6,7]. The flow in the Penn State combustor has been modeled as the turbulent flow of compressible reactive fluid in an axisymmetric problem formulation. The simulations were performed on a Dell T7500 workstation with an Intel Xeon E5645 processor (six cores, and 12 threads).

\section{A. Computational Domain}

The employed CFD code uses the finite volume element method. The numerical simulations of the flow inside the chamber have been carried out in a quasi-three-dimensional domain, which represents a sector of $1 \mathrm{deg}$ and one element thick. The domain includes the combustion chamber, the converging-diverging nozzle, and a small part of the coaxial injector. The domain is shown in Fig. 1. The axisymmetric combustion chamber has a diameter of $38.1 \mathrm{~mm}$ (1.5 in.). The length of the cylindrical part is $285.75 \mathrm{~mm}$. The throat diameter is $8.166 \mathrm{~mm}$. The thickness of the injector post (the thickness of wall between the fuel and oxidizer in the coaxial injector) is $0.52 \mathrm{~mm}$, whereas the gap in the outer annulus is $0.595 \mathrm{~mm}$.

Although turbulent flow is always three-dimensional, the RANS equations do not require three-dimensional settings if the imposed boundary conditions are two-dimensional. The use of the 2-D numerical domain allowed the number of elements in the mesh to be significantly reduced. The reduced mesh size decreased the computation time to several hours compared to 3-D simulations. In the previous study [12], it was found that, for the flame of a coaxial injector, the simulation results depended on the velocity profile in the (outer) annular passage of coaxial injector and that the uniform velocity profile resulted in a wrong spreading angle and poor convergence at the grid nodes near the oxygen post tip. Therefore, the numerical domain included a small part of the outer annular passage. Due to the small gap in the annular passage $(h / L<0.1)$, this small part was sufficient for getting a U-shaped velocity profile at the outlet of the injector.

The simulations were performed on hexahedral structured meshes with mesh refinement near the walls and in the flame region. The numerical meshes were generated using the computer program ICEM from the package ANSYS CFD. Following previous experience in CFD simulations [10,12], the generated meshes were refined near the injector post and around the shear layer between the jets of the fuel and oxidizer; here, the spacing reaches $15 \mu \mathrm{m}$. In a base mesh, the spacing between the nodes varies from $0.6 \mu \mathrm{m}$ to $0.5 \mathrm{~mm}$, and most of the mesh elements are stretched in the axial direction. The smallest spacing is located on the sidewalls, but the coarsest part of the mesh is located in the diverging part of the nozzle. Prism layers near the walls grow with the expansion ration of 1.1. In the whole domain, the expansion ratio does not exceed a value of 1.2. The base mesh consists of 1.5 million nodes.

An unstructured hex-dominant mesh was also generated and tested. The unstructured mesh had the same mesh resolution near the 


\section{Gas from}

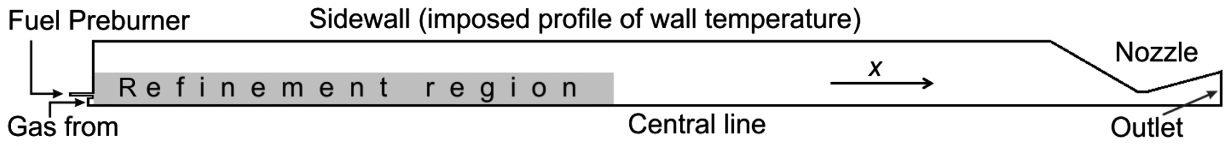

Oxidizer Preburner

Fig. 1 Computational domain and boundary conditions.

walls and in the refinement region as the structured mesh but contained more nodes. This mesh has given the same solution as the structured mesh but also required more computational power due to the larger mesh size.

\section{B. Turbulence Model}

The flow in the combustion chamber has been modeled as a steadystate solution of the Favre-averaged Navier-Stokes equations. The equations have been solved using a second-order advection scheme. Turbulence has been modeled using the SST turbulence model with the default values of the coefficients and the "automatic" near-wall treatment [7]. When using the automatic near-wall treatment, the solver switches from a wall-function formulation to a low-Reynoldsnumber near-wall formulation when the mesh is refined. The turbulent transport has been modeled using the default value of 0.9 for the turbulent Schmidt number. However, the final results have been obtained by assigning the turbulent Schmidt number of 0.7 . The value of 0.7 has been recommended for the turbulent Schmidt number in axisymmetric turbulent free round jets by Yimer et al. [13]. The turbulent Prandtl number has been set to the default value of 0.9 , which has been extensively validated for heat transfer predictions in CFX [14].

\section{Model of a Non-Premixed Turbulent Flame}

The turbulent combustion of propellants in a non-premixed flame has been modeled using the eddy-dissipation-type model [11]. The employed model is based on the assumption of a thin flame (i.e., chemical reactions are infinitely fast), and the rate of chemical transformations is limited by the mixing rate. In the used model, chemical transformations occur in a single-step global reaction

$$
\mathrm{H}_{2}+0.5 \mathrm{O}_{2} \rightarrow \mathrm{H}_{2} \mathrm{O}
$$

in which the reaction rate is proportional to the rate of eddy dissipation:

$$
\text { Reaction rate } \propto \epsilon / k
$$

where $\epsilon$ is the turbulence eddy dissipation, and $k$ is the turbulent kinetic energy. The assumptions, on which the model is based, hold up well in rocket combustion chambers operated with hydrogen. Hydrogen and oxygen react immediately at high temperatures and pressures. Since the propellants are injected separately in a combustion chamber, the combustion process is limited by the mixing. This statement clearly follows from the study by Ivancic and Mayer [15]. They evaluated timescales in a cryogenic hydrogenoxygen subscale chamber and found that, at high pressures, the chemical timescale is smaller by several orders of magnitude than the Kolmogorov timescale. In particular, for the Penn State test case, Yang also evaluated chemical and Kolmogorov timescales and found in [3] that the chemical timescale is over two times smaller than the Kolmogorov timescale.

The advantages of the eddy-dissipation model (EDM) are its simplicity and robustness, but to achieve acceptable results, the model should be extended, especially for the case of rocket combustion. At high temperatures $(T>3000 \mathrm{~K})$, the dissociation of $\mathrm{H}_{2} \mathrm{O}$ becomes important, namely, the fraction of $\mathrm{H}_{2} \mathrm{O}$ in a chemical equilibrium mixture is significantly less than $100 \%$ in burned gases in rocket engines. The direct use of Eq. (1) gives a flame temperature near $5000 \mathrm{~K}$, whereas the flame temperature in rocket combustion chambers amounts to around $3500 \mathrm{~K}$. To predict the flame temperature accurately, it is necessary to take into account the incomplete combustion of hydrogen. To obtain the correct flame temperature in the combustion chamber, which is very important for the heat balance of the combustion chamber and the predictions of the heat fluxes, an external parameter (called "maximum flame temperature" in CFX [6] ) has been used in the model. The reaction rate is set to zero when the temperature of reactive mixture reaches the value of the Maximum flame temperature, which is precalculated using the program NASA Chemical Equilibrium with Applications (CEA) [16].

Another important process in a flame, which should be taken into account in modeling, is flame extinction. At ordinary conditions, first of all, it is necessary to take into account the flame extinction outside flammability limits. Even for hydrogen-oxygen rocket engines, flammability limits are an important issue because one or both components are injected at cryogenic temperatures. In the Penn State test case, the propellants are injected at high temperatures (in contrast to cryogenic engines). For this reason, the flammability limits are not included in the present model.

The present model has two additional parameters for the accurate modeling of the interaction between flame and turbulence. The turbulent mixing rate $\epsilon / k$ becomes large close to walls due to the drop of $k$. Therefore, the value of $\epsilon / k$ in Eq. (2) is limited to a value of $10^{4} \mathrm{~s}^{-1}$, which is set by a parameter called the "mixing rate limit" in CFX [6]; otherwise, the reaction rate goes up unnaturally near walls. At a certain level of turbulence, the dissipation of heat and radicals leads to flame quenching. In regions of high turbulence, when the turbulence mixing timescale $k / \epsilon$ is smaller than a chemical timescale, a local extinction occurs in the present model, i.e., the reaction rate is set to zero. The chemical timescale is defined in the model as a ratio of the laminar flame thickness to the laminar flame velocity. The flame thickness is evaluated using Blint's correlation [17], whereas the laminar flame velocity is calculated using the hydrogen kinetic mechanism of Burke et al. [18] and the computer code PREMIX [19], which is the part of the software package CHEMKIN.

In contrast to the standard formulation of the EDM model in CFX, all parameters describing the flame (maximum flame temperature, mixing rate limit, and chemical timescale) are not constants in the present model but are instead the functions of the mixture fraction, which is the total mass fraction of the hydrogen element in mixture and plays the role of equivalence ratio in the calculations.

\section{Model of Thermodynamic Properties}

The model of the thermodynamic properties of gas in a combustion chamber has a significant impact on the simulation results. Because the propellants are injected at relatively high temperatures, all components of the gas mixture can be treated as ideal gases. However, some properties of the gas mixture cannot be easily defined from the properties of its components.

The enthalpy and the entropy of the individual components have been defined using NASA polynomials [20]. The dynamic viscosity and thermal conductivity of the mixture components have been defined using Sutherland's law with parameters recommended in [21] in order to take into account the increase of the thermal conductivity and viscosity of gases with temperature.

CFX defines the property of multicomponent mixture using a mass averaging, by which the contribution of the individual components to the properties of the mixture is directly proportional to the mass fraction. This leads to the transport coefficients for the mixture of hydrogen, with oxygen being underestimated. Hydrogen has very high thermal conductivity; its presence significantly increases the thermal conductivity of the mixture. The mass averaging completely 
eliminates the influence of hydrogen on the transport properties of a mixture; for example, in an equimolar mixture of hydrogen and oxygen, the hydrogen mass fraction equals to $6 \%$, so the contribution of hydrogen into the mixture properties amounts to only $6 \%$ according to the mass averaging. The inadequacy of the mass averaging clearly follows from the data of $[22,23]$, where the experimental data on the viscosity and thermal conductivity of $\mathrm{H}_{2}-\mathrm{O}_{2}$ mixtures at standard conditions have been presented. For this reason, the transport properties of the gas mixture have been modeled separately using the CFX Expression Language [6] by empirical formulas suggested in [24]. The effect of the new mixing rule is especially marked in the thermal conductivity and reaches $100 \%$ when hydrogen mass fraction amounts to from 2 to $20 \%$.

\section{Mesh Convergence Study}

The flow in a rocket combustion chamber is characterized by the presence of a large amount of different scales. Under such circumstances, it is essential to perform a mesh convergence study in order to resolve all necessary scales and obtain a mesh-independent solution. The mesh convergence study should be carried out even if simulations are performed using the RANS equations. Time averaging eliminates many scales; however, the scales associated with boundary conditions remain.

In the current work, the mesh study has been performed by a successive uniform refinement of the mesh. During the refinement, the spacing was reduced twofold in the axial and radial directions. In addition, the structure of the mesh (i.e., blocking) was kept the same. It is necessary to note that the initial coarse mesh already had

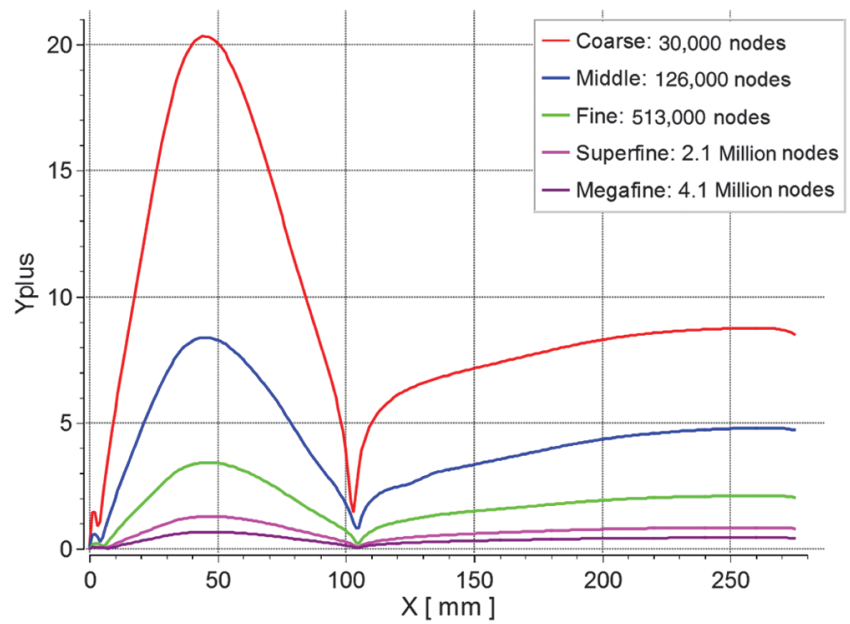

Fig. 2 Near-wall resolution of different meshes during the mesh convergence study.

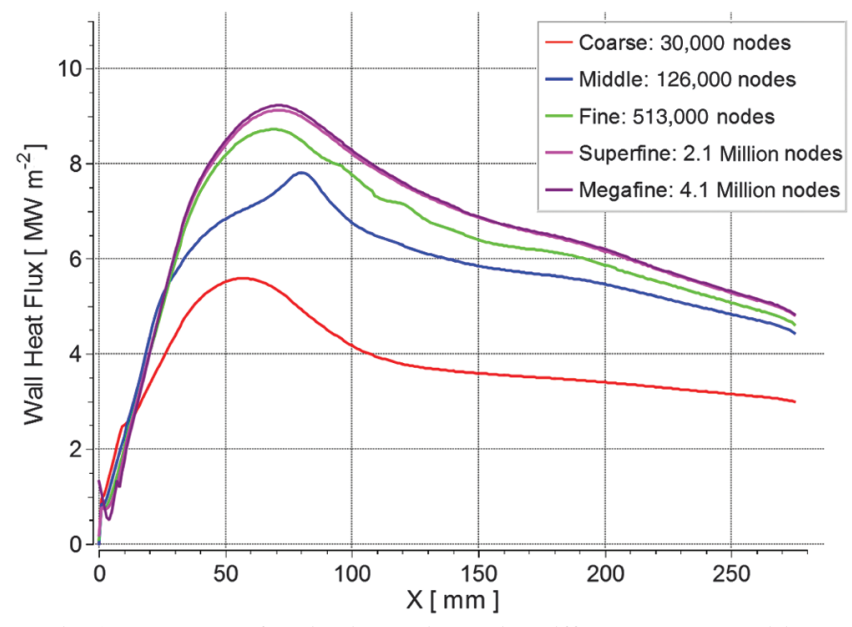

Fig. 3 Wall heat flux in simulations with different mesh densities. refinement around the sidewalls, the injector post, and in the shear layer between the jets of fuel and oxidizer. In CFD simulations, the accurate prediction of wall heat fluxes requires high mesh resolution near the walls. For this reason, the near-wall grid resolution was improved approximately three times at each step refinement (i.e., with a higher rate than the mesh itself). The process of the grid refinement during the mesh convergence study is illustrated in Fig. 2 . The amount of mesh points was increased four times at each step from $30 \times 10^{3}$ nodes to $4.1 \times 10^{6}$ nodes. The near-wall resolution was improved during the grid convergence study in terms of maximum $y^{+}$ from 20 to 0.7 , where $y^{+}$is the dimensionless distance from the wall [7]. At the last step, the spacing was reduced by only a factor of 1.4 , and the near-wall resolution was improved by a factor of 2 .

The mesh convergence study has shown that the solution has become practically mesh independent in the volume of the chamber after the second refinement, i.e., at the mesh resolution of the "fine" mesh. However, for the used near-wall treatment, the mesh with $y^{+}$ around one is recommended for accurate heat transfer predictions [7]. The wall heat fluxes obtained in simulations with meshes of different densities are shown in Fig. 3 . As one can see, the simulated wall heat flux goes up with the increase of near-wall resolution and becomes grid independent at the level of $y^{+} \approx 1$. Hence, the mesh-independent solution has required further mesh refinement and been obtained with the "superfine" mesh, which has a near-wall resolution of $y_{\max }^{+}=1.4$. The difference between the results obtained with the superfine mesh and with the "megafine" mesh, which has twice as many nodes and $y_{\max }^{+}=0.7$, is so small that the lines corresponding to the superfine and megafine meshes plotted in Fig. 3 overlap.

Since it is possible to obtain the mesh-independent solution in the volume of the combustion chamber with a mesh coarser than the superfine mesh, the final mesh was generated with a new nodal distribution and block structure but with the same near-wall resolution as the superfine mesh. The final mesh consists of 1.5 million nodes and has a near-wall resolution of $y^{+} \approx 1$. The final mesh is not presented in Figs. $2 \underline{2}$ and $\underline{3}$, because it gives a solution coinciding with the solution on the superfine mesh, and the lines corresponding to the final mesh are indistinguishable from the lines of the superfine mesh on the graphs. The new mesh has allowed a $13 \%$ reduction in computational time relative to the superfine mesh.

\section{A. Analysis of Numerical Model}

The mesh convergence study has been done using the basic models and settings of CFX. The wall heat flux obtained during the mesh convergence study appeared significantly lower than the experimental data (even on the very fine meshes). In the experiment, the wall heat flux reaches a maximum of around $16 \mathrm{MW} / \mathrm{m}^{2}$ [1], whereas in the simulation, the maximum wall heat flux is about $9 \mathrm{MW} / \mathrm{m}^{2}$. For this reason, we have developed our own model of reactive flow in rocket combustion chambers.

Combustion in a liquid rocket engine is characterized by many processes. The detailed modeling of all processes is challenging and requires great effort. Performing CFD simulations at different complexity levels of modeling, the effect of the models on the simulation results was studied, and it was found that a minimum modeling level was required for accurate predictions of wall heat fluxes. In the present study, the complexity of the numerical model was gradually increased within eight steps from the simplest models and settings up to the inclusion of a radiation heat transfer model at the final step; see Table 3 . The results of numerical simulations at the different depths of modeling are shown in Fig. 4.

\section{Steps 0 and 1}

Step 0 represents the "lowest" level of modeling. At this level, the basic models and settings of CFX have been used for the simulation. These results coincide with the results obtained during the mesh convergence study with the use of the superfine mesh, and the difference is only in the use of the final mesh instead. At the next step (step 1), the EDM combustion model has been modified, as has been previously described in Sec. II.C. The additional model parameters 
Table 3 Brief summary of model modifications from step 0 to step 8

\begin{tabular}{lcc}
\hline \hline Step & Description & $\begin{array}{c}\text { Main common } \\
\text { features }^{\mathrm{a}}\end{array}$ \\
\hline 0 & Default parameters of CFX & I \\
1 & Extended EDM model & I \\
2 & Sutherland's equations for viscosity and thermal & II \\
& conductivity & II \\
3 & Symmetry boundary conditions at side planes & II \\
4 & $k-\omega$ turbulence model (only step 4) & II \\
5 & Wall roughness is set to 5 $\mu \mathrm{m}$ & III \\
6 & "New" mixing rule for transport coefficients [24] & III \\
7 b & Value of the turbulent Schmidt number is changed & \\
& from 0.9 to 0.7 & III \\
8 & Radiation heat transfer: the P1 model & \\
\hline \hline
\end{tabular}

aDefault CFX values for transport properties denoted by I, Sutherland's equations for viscosity and thermal conductivity denoted by II, and new mixing rule for transport coefficients [24] denoted by III.

${ }^{\mathrm{b}}$ Taken as the final results.

slightly change the shape of the flame. This can be seen in Fig. 5 , where the simulations at three different modeling levels (including step 0 ) are presented. The new combustion model results in a slightly longer flame and in a lower temperature near the injector post. However, the effect of the combustion model on wall heat flux is insignificant. The main benefit of the developed extension of the EDM model is an improved convergence. The reaction rate in the vicinity of the injector post is lower in the new model, and the spatial distribution of reaction rate across the chamber is more uniform. The modifications of the EDM model have resulted in a decrease of the residuals on average from $10^{-5}$ to $10^{-6}$. However, the tight convergence was not a main objective of the developed model. The applied combustion model has been developed for the modeling of the flame of cryogenic propellants, so its advantages over the standard EDM model are not visible in this particular case.

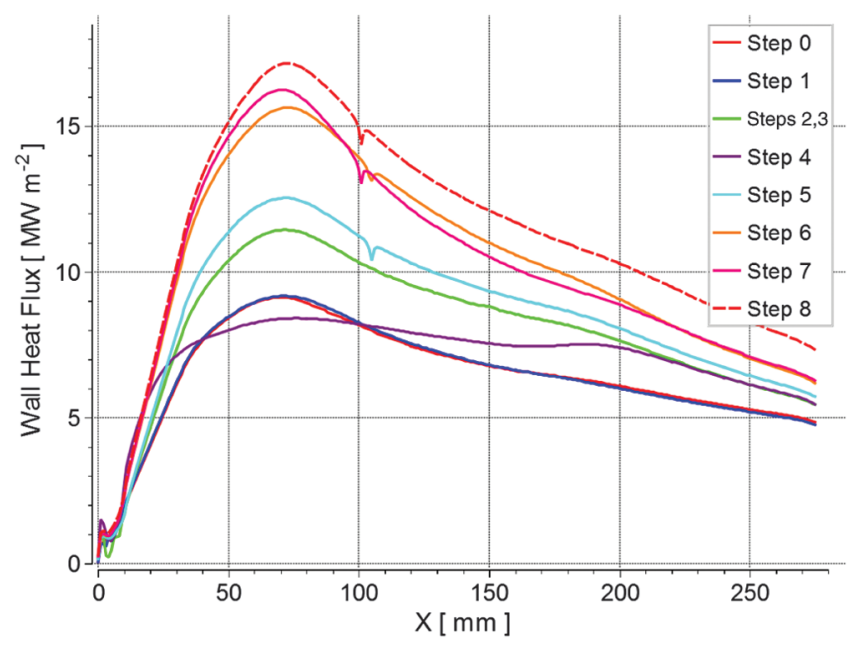

Fig. 4 Results of CFD simulations at different modeling levels.

\section{Step 2}

At the following step, the temperature-dependent transport coefficients for individual mixture components have been introduced. By default, in CFX (steps 0 and 1), constant transport coefficients have been used. They are not adequate in the case when the temperature varies in a wide range because the transport coefficients increase as the square root of temperature. That is why the Sutherland's equations give higher values of transport coefficients at higher temperatures than default values of CFX. This results in the higher values of wall heat fluxes at step 2. As one can see in Fig. 4, the CFD model at this step predicts the substantially higher wall heat flux.

\section{3. $\quad$ Step 3}

The switch of the boundary conditions at the side planes from periodic boundary conditions to symmetry boundary conditions (step 3 ) does not lead to any changes of simulation results; however, it has improved the convergence rate of the numerical model more than tenfold. In Fig. 4, the line corresponding to step 3 matches the curve of step 2 completely. It was necessary to perform this test because symmetry boundary conditions impose additional constraints on the flow and may affect simulation results in some configurations.

\section{Step 4}

Step 4 shows the impact of turbulence model. At this step, the $k-\omega$ turbulence model was used in the simulation instead of the SST turbulence model. The SST model is based on the $k-\omega$ turbulence model. With the use of blending functions, the SST model switches to the $k$ - $\epsilon$ turbulence model in free shear flows; however, in near-wall regions, the $k-\omega$ and SST models have the same formulation [7]. The $k-\omega$ turbulence model results in a slightly longer flame and lower temperatures in the recirculation zone and in the outside areas of the flame (Fig. 5), which gives the lower wall heat flux; see Fig. 4 . The comparison of the results obtained using the SST and $k-\omega$ models (step 3 and step 4) shows that the wall heat flux also depends on the flow in the middle of the chamber and the turbulence model in this area. The SST model has been used in all subsequent steps again.

\section{5. $\quad$ Step 5}

Surface properties and boundary conditions at the walls have a profound effect on wall heat flux. Even if chamber walls have been polished before the hot test, they become eroded after the first seconds of the hot-fire test. At step 5, the boundary conditions at the walls were switched from smooth walls to rough walls. The wall roughness has been set to $5 \mu \mathrm{m}$ based on previous experience in combustion chamber testing. The switch to rough walls increased the maximum wall heat flux by approximately $1 \mathrm{MW} / \mathrm{m}^{2}$. At the same time, a kink appeared in the heat flux profile at the stagnation point, which is probably an artifact of the employed near-wall treatment.

\section{Step 6}

The next step emphasizes the validity of the conclusion of past work about the unsuitability of the mass averaging for the evaluation of the transport properties of hydrogen-oxygen mixtures in combustion simulations [25]. For step 6, and all subsequent steps, the empirical formulas for viscosity and the thermal conductivity of the gas mixture have been applied instead of the mass-averaging

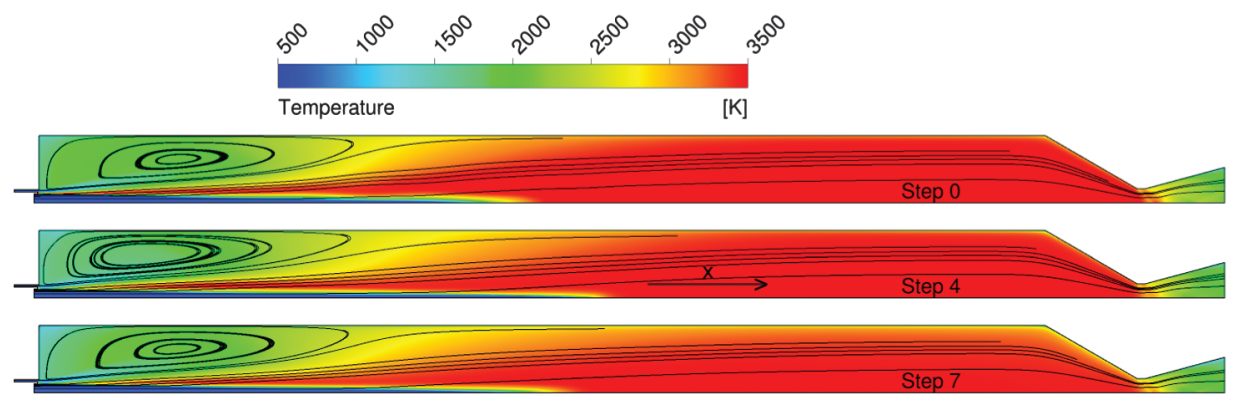

Fig. 5 Results of CFD simulations at different modeling levels. 
approach used in CFX by default. The mass averaging eliminates the impact of hydrogen on transport properties due to its low molecular weight. The abandonment of the mass averaging for viscosity and thermal conductivity has given a substantial increase of the simulated wall heat flux. The effect has been achieved through the increase of the thermal conductivity in the boundary layer at the wall. The detailed information about the modeling of transport properties can be found in Sec. II.D of this paper.

\section{7. $\quad$ Step 7}

Step 7 is also related to the transport properties but in turbulent flow. The turbulent Schmidt and Prandtl numbers, which are responsible for turbulent diffusion and thermal conductivity, respectively, are set to 0.9 by default in CFX. At this step, the turbulent Schmidt number was set to 0.7, as was suggested in [13]. The change in the value of the turbulent Schmidt number leads to a slightly better agreement with the experiment; however, in contrast to the work of Ivancic et al. [5], step 7 is not an "adaption of the model settings." The change of the value of the turbulent Schmidt number was done because the value of 0.7 was also used by the author in all other CFD simulations of hydrogen rocket combustion chambers for the last two years. The change of the turbulent Schmidt number results in slightly different distributions of oxygen and temperature in the combustion chamber.

\section{8. $\quad$ Step 8}

For the final step, the numerical model has been complemented with the model of radiation heat transfer. For this purpose, the P1 model [7] has been employed in accordance with the recommendations of $\mathrm{CFX}$ [6]. The conditions in the combustion chamber model meet the model requirements, i.e., the optical thickness is large. As we can see in Fig. 4, the radiative heat transfer substantially increases the wall heat flux. It should be noted that the results of the calculations depend strongly on the optical characteristics of the surface. The emissivity of copper varies more than 10 times from polished copper to oxidized (at room temperature, from 0.02 to 0.6 , respectively). In the simulations at step 8 , the surfaces of the chamber walls were assumed to be coated by an oxide layer but clean from oxide scale. However, the walls of rocket combustion chambers do not always become oxidized after hot firing. If there is an excess of hydrogen in the gas mixture, then hydrogen reduces the metals from oxides and protects chamber walls from the oxide film formation. If the walls are not oxidized during the hot-fire test, then the effect of the radiative heat transfer is negligible. In the considered test case, the measurements have been performed at an overall ROF of 6.6 (see Table 2), which corresponds to the excess of hydrogen in the mixture. At the same time, Pal et al. [1] did not provide any information about the condition of the walls during or after the hotfire test. Taking into account that the walls were probably not oxidized during the experiment, the results of step 7 (i.e., without the radiation model) are considered as the final results of the current work. The idea of performing step 8 is to study the potential effect of the radiative transfer on the heat flux.

The gradual increase of model complexity has resulted in the increase of the wall heat flux. The main effect has been given by the increase of the transport coefficients at step 2 and step 6 . Thus, the predicted wall heat fluxes depend primarily on the model of the thermodynamic properties and, particularly, on the thermal conductivity of gas in the boundary layer. The effect of molecular thermal conductivity on the wall heat flux is easily explained. The largest temperature drop toward the wall is located in the laminar sublayer. Thus, the laminar sublayer is a bottleneck for the wall heat flux. Therefore, the thermal conductivity in the laminar sublayer has a significant impact on the wall heat flux.

The combustion and turbulence models also matter in the CFD simulations. In the studied case, they are responsible for the shape of the flame and recirculation zone. For example, the switch from the SST turbulence model to the $k-\omega$ model in step 4 led to the change of streamlines near the stagnation point, and that resulted in the significant decrease of the maximum wall heat flux (Fig. 4). The impact of combustion and turbulence models on wall heat flux in the Penn State test case was studied in detail in the work of Ivancic et al. [5]. They compared the numerical results obtained with the use of eight different turbulence models and found that some of the models predicted completely different shapes of the main recirculation zone and, accordingly, different wall heat profiles. Ivancic et al. [5] also compared different combustion models. In their simulations, different combustion models predicted different temperatures of gas mixtures in the combustion chamber. As a result, the effect of the combustion model is visible on the wall heat flux profile [].

\section{B. Comparison with Experiment and Other Works}

Before comparing the simulation results with the experimental data and other simulations, it is necessary to identify the experimental uncertainty. Unfortunately, the authors of the experiment did not provide any information about the experimental uncertainty; however, earlier, they had presented a more detailed description of similar measurements in the Penn State combustor [2]. The value of the experimental uncertainty in the Penn State test case may be evaluated from the results of [2], where the same experimental setup and method were used. The results of the current analysis of the experimental uncertainties have been compiled in Table 4 . The aim of the analysis is to evaluate an expected difference between the experimental data and the numerical results obtained with the use of an ideal CFD model and the boundary conditions presented in the original work [1].

Firstly, the experimental data are characterized by a statistical error. Generally, all parameters in rocket combustion chambers (pressure, temperature, etc.) oscillate. (Sometimes during hot-fire tests, oscillations reach $100 \%$ of the measured value.) Marshall et al. [2] presented a time profile of the wall heat flux where the random oscillation of the wall heat flux amounted to $8 \%$ of the measured value.

The next contributing factors are the conditions at the walls. During hot-fire tests, chamber walls are exposed to erosion. Even if the combustion chamber walls are polished before the hot test, after a short period of operation, they become rough. The erosion and oxidation traces after hot tests are always distributed nonuniformly. The distribution depends strongly on local conditions at the walls. The oxidation and erosion of the wall surface have a significant impact on the wall heat flux. The comparison of the curves for step 3 and step 5, and for step 7 and step 8, in Fig. 4 shows the impact of the wall roughness and the wall emissivity on the wall heat flux. The average difference between step 7 and step 8 amounts 10\%, which corresponds to the difference between unoxidized and fully oxidized walls. The real conditions are somewhere in between; thus, the contribution of radiative transfer into experimental error is estimated to be 5\%. As for the wall roughness, the difference between step 3 and step 5 does not correspond to the whole span of possible boundary conditions. The value of $5 \mu \mathrm{m}$ for the wall roughness is not the upper margin but the conservative estimate of the wall roughness during the experiment. Thus, the contribution of the wall roughness has been estimated as equal to the difference between step 3 and step 5, which amounts to $8 \%$.

The last (but not least) important factor is the uncertainty of the injection boundary conditions. This problem was already analyzed in the work of Ivancic et al. [5] and mentioned in the Introduction (Sec. I). The problem has two sides. First, the temperatures of gases injected into the main chamber are significantly lower than the

Table 4 Contribution of different factors into experimental uncertainty

\begin{tabular}{lcc}
\hline \hline Factor & Uncertainty, \% & $\begin{array}{c}\text { Contribution } \\
\text { to error, \% }\end{array}$ \\
\hline Statistical error & 8 & 30 \\
Conditions at walls (wall roughness) & 9 & 40 \\
Conditions at walls (wall emissivity) & 5 & 10 \\
Injection boundary conditions & 7 & 20 \\
\hline Estimated experimental error & 15 & 100 \\
\hline \hline
\end{tabular}


temperatures calculated with the assumption of complete combustion in the preburners. The inconsistency is as follows: the assumption of complete combustion was used for the calculations of the composition of the fuel-rich gases with the use of the NASA CEA program [16] by $\mathrm{Pal}$ et al. [1]. These calculations gave the temperature of the fuel-rich gases of $1360 \mathrm{~K}$, whereas the specified (measured) temperature of the fuel-rich gases is $811 \mathrm{~K}$. Pal et al. have explained this discrepancy by the heat loss to the preburner walls, although such a loss would amount to $35 \%$ of the heat loss in the main chamber. At the same time, it is necessary to take into account that the preburner and the main combustion chamber have a similar design, but the temperature in the main chamber is significantly higher: around $3500 \mathrm{~K}$. Therefore, the large heat losses in the preburners do not sound realistic. The more realistic explanation would be a flow stratification in the preburners so that the temperature in the middle of the preburners is higher than on the periphery.

The second side of the problem is that the pressure of 54.2 bar measured in the main combustion chamber significantly exceeds the pressure calculated using the inlet boundary conditions stated in the original work [1]; see also Table 2. The simple estimation of the pressure in the combustion chamber can be done with the use of CEA [16]. The calculations on the assumptions of the ideal rocket combustion chamber result in a pressure of 51.7 bar in the Penn State combustor; the account for the heat loss reduces the pressure to 48.6 bar. (The NASA CEA code gives the low estimate of the pressure in the case of heat losses due to the assumption of the uniform flow in the nozzle.) The CFD simulations give a more accurate estimation of the pressure of 50 bar in the combustion chamber. The difference between the measured and simulated pressures can serve as the estimation of the uncertainty of the inlet boundary conditions (mixture composition and temperature). Because the wall heat flux correlates with the pressure in the Penn State combustor $\left(q_{w} \propto p^{0.8}\right)$ [2], the contribution of the uncertainty of the inlet boundary conditions to the relative uncertainty of the wall heat flux can be calculated as follows:

$$
\begin{aligned}
\Delta q_{w} / q_{w} & =0.8 \times\left(p_{\exp }-p_{\text {sim }}\right) / p_{\text {sim }} \\
& =0.8 \times(54.2 \text { bar }-50.0 \text { bar }) / 50.0 \text { bar } \approx 7 \%
\end{aligned}
$$

Most of the factors presented in Table 4 are related to the uncertainty of the boundary conditions. They were added to the experimental error because measurement results cannot be considered in isolation from boundary conditions. The uncertainties of the boundary conditions are the results of the first-order approximations and have a probabilistic character. For this reason, the uncertainties of the boundary conditions have been treated in the same manner as statistical errors. All aforementioned uncertainties are independent; thus, the cumulative experimental error equals to the square root of the sum of the squares of the single components

$$
\text { Error }_{\text {tot. }}=\sqrt{\sum \text { Err. }_{i}^{2}}
$$

and amounts to $15 \%$. The margins of $15 \%$ are plotted with the experimental data in Fig. $\underline{6}$, but it is necessary to remember that these margins are in fact the standard deviations, which means that the real value of heat flux can also be located outside the margins with the probability of $32 \%$. At the same time, the error margins in Fig. 6 do not contain the systematic error and other unknown uncertainties of the measurements (uncertainties of flow rates and heat flux itself), so the real experimental error may significantly exceed these by $15 \%$. In particular, the used experimental method requires a good thermal contact between thermocouples and chamber walls; otherwise, the bias from the real heat flux is significant. The derived value of the experimental error is indeed a conservative estimate, and it would be reasonable to consider the total experimental error equal to around $20-25 \%$.

The current numerical results (step 7) agree well with the experimental data and the CFD results of other groups; see Fig. 6. All simulations compared in Fig. 6 have been obtained with the use of the RANS equations and correlate with each other very well. However,

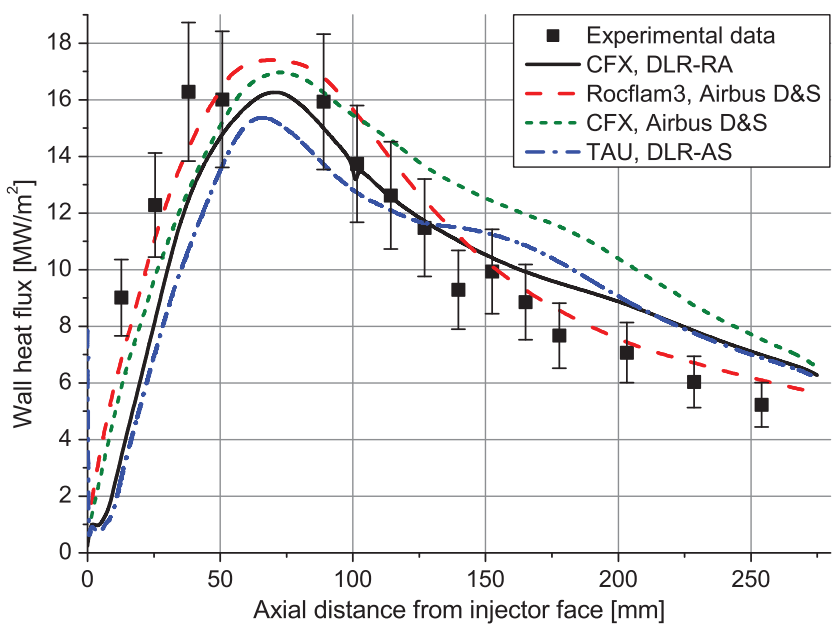

Fig. 6 Comparison of the current results (black line) with experimental data [1] and simulation results of other groups [].

the results obtained with the use of the CFD code Rocflam 3 have excellent agreement with the experimental data, which are the result of the fitting of the turbulent Schmidt and Prandtl numbers. The other simulations lie below the experimental points and outside the margins of the experimental error at $x<50 \mathrm{~mm}$, and they lie above the experimental points at $x \geq 175 \mathrm{~mm}$. Unfortunately, the comparison of the different simulations does not allow us to make any conclusions, except for the fact that it is possible to predict wall heat fluxes in rocket combustion chambers using RANS simulations. All four simulations presented in the figure have been performed using different turbulent and combustion models, different turbulent Prandtl and Schmidt numbers, and slightly different boundary conditions. This is the reason why it is so difficult to draw conclusions from the comparison of the different CFD simulations. Even the comparison of the results obtained with the use of the same code (CFX) has not given the opportunity to explain the difference in the results. There are also other CFD simulations of the Penn State combustor (e.g., [3,4], and many others) performed with the use of RANS, URANS, detached-eddy simulation, and LES; but, as we can see, even the comparison of the similar CFD models may be uninformative.

\section{Conclusions}

In the present work, the Penn State test case has been simulated with the use of the RANS equations. The target of the simulations has been the measured wall heat fluxes in the staged hydrogen-oxygen combustor operating at 54 bar. To model turbulent non-premixed flames in rocket engine combustion chambers, the new EDM-type model has been developed. The developed model has the additional parameters: maximum flame temperature, mixing rate limit, and chemical timescale. The distinctive feature of the new model is that these parameters are not constants and depend on the local mixture composition. Another essential feature of the numerical model is the model of the thermodynamic properties where the transport properties of the gas mixture have been defined without the use of the mass averaging. To eliminate the effect of the spatial discretization, a mesh convergence study has been conducted, and a meshindependent solution was obtained.

The impact of the different elements of the numerical model has been studied. The analysis of the numerical results obtained at the different levels of model complexity showed that the wall heat flux predictions depend strongly on the model of the transport properties.

The experimental uncertainties of the test case have been analyzed using the information and data from a similar experiment by the same group. The uncertainty of the wall heat flux measurements has been estimated and amounts to $15 \%$.

In the current work, the predicted wall heat fluxes agree very well with the experimental data. Good agreement with the experimental data was also achieved in other RANS simulations. However, this does not prove that the RANS equations are an ultimate tool for 
modeling the flow in rocket combustion chambers. Turbulent flow can be simulated with the use of the RANS equations and statistical turbulence models when the flow is statistically stationary and the turbulence does not require the representation of many scales; otherwise, LES or LES-like simulations are required. Wall heat fluxes depend mainly on the flow near the walls. The RANS equations and the SST turbulence model can adequately model the attached flow near walls and the recirculation zone behind a back step. For these reasons, good agreement with the experimental data has been achieved in the Penn State test case, and that is why the RANS equations may be used for the predictions of wall heat fluxes.

\section{Acknowledgments}

The author thanks the staff members of ANSYS Germany: Katya Georgieva-Angelova for helpful discussions, and Jill Ellegast and Bae-Hong Chen for their help in the mesh generation. The author is also grateful to Scott Beinke at the German Aerospace Center in Lampoldshausen for valuable comments that improved the paper. Most of the results of this work were presented at the scientific conference Space Propulsion 2014, in Cologne, Germany, 1922 May 2014.

\section{References}

[1] Pal, S., Marshall, W., Woodward, R., and Santoro, R., "Wall Heat Flux Measurements for a Uni-Element $\mathrm{GO}_{2} / \mathrm{GH}_{2}$ Shear Coaxial Injector," Third International Workshop on Rocket Combustion Modeling, Snecma, Safran Group, Vernon, France, March 2006.

[2] Marshall, W., Pal, S., Woodward, R., and Santoro, R., "Benchmark Wall Heat Flux Data for a $\mathrm{GO}_{2} / \mathrm{GH}_{2}$ Single Element Combustor," AIAA Paper 2005-3572, July 2005.

[3] Tucker, P. K., Menon, S., Merkle, C. L., Oefelein, J. C., and Yang, V., "Validation of High-Fidelity CFD Simulations for Rocket Injector Design," AIAA Paper 2008-5226, July 2008.

[4] Sozer, E., Vaidyanathan, A., Segal, C., and Shyy, W., "Computational Assessment of Gaseous Reacting Flows in Single Element Injector," AIAA Paper 2009-0449, Jan. 2009.

[5] Ivancic, B., Riedmann, H., Frey, M., Knab, O., Karl, S., and Hannemann, K., "Investigation of Different Modeling Approaches for CFD Simulation of High Pressure Rocket Combustors," 5th European Conference for Aerospace Sciences, CIMNE, Barcelona, July 2013.

[6] ANSYS CFX-Solver Modeling Guide, Release 13.0, ANSYS, Inc., Canonsburg, PA, 2010.

[7] ANSYS CFX-Solver Theory Guide, Release 13.0, ANSYS, Inc., Canonsburg, PA, 2010.

[8] DLR TAU Code [online database], DLR, German Aerospace Center, Inst. of Aerodynamics and Flow Technology, http://tau.dlr.de/ [retrieved 5 June 2014].

[9] Durbin, P. A., "On the $k-\epsilon$ Stagnation Point Anomaly," International Journal of Heat and Fluid Flow, Vol. 17, No. 1, 1996, pp. 89-90. doi:10.1016/0142-727X(95)00073-Y

[10] Zhukov, V. P., and Suslov, D., "Wall Heat Fluxes in Rocket Combustion Chamber with Porous Injector Head," 5th European Conference for Aerospace Sciences, CIMNE, Barcelona, July 2013.
[11] Magnussen, B. F., and Hjertager, B. H., "On Mathematical Models of Turbulent Combustion with Special Emphasis on Soot Formation and Combustion," Symposium (International) on Combustion, Vol. 16, No. 1, 1977, pp. 719-729. doi:10.1016/S0082-0784(77)80366-4

[12] Zhukov, V. P., and Feil, M., "Numerical Simulations of Flame of Single Co-Axial Injector," 5th European Conference for Aerospace Sciences, CIMNE, Barcelona, July 2013.

[13] Yimer, I., Campbell, I., and Jiang, L.-Y., "Estimation of the Turbulent Schmidt Number from Experimental Profiles of Axial Velocity and Concentration for High-Reynolds-Number Jet Flows," Canadian Aeronautics and Space Journal, Vol. 48, No. 3, 2002, pp. 195-200. doi: $10.5589 / \mathrm{q} 02-024$

[14] Vieser, W., Esch, T., Menter, F., and Smirnov, P., "Heat Transfer Predictions Using Advanced Two-Equation Turbulence Models," CFX Validation Rept., ANSYS Germany, Otterfing, Germany, 2007.

[15] Ivancic, B., and Mayer, W., "Time- and Length Scales of Combustion in Liquid Rocket Thrust Chambers," Journal of Propulsion and Power, Vol. 18, No. 2, 2002, pp. 247-253. doi: $10.2514 / 2.5963$

[16] McBride, B. J., and Gordon, S., "Computer Program for Calculation of Complex Equilibrium Compositions and Applications," NASA Reference Publ. 1311, 1996.

[17] Poinsot, T., and Veynante, D., "Premixed Flame Thicknesses," Theoretical and Numerical Combustion, Edwards, Philadelphia, 2001, pp. 56-58.

[18] Burke, M. P., Chaos, M., Ju, Y., Dryer, F. L., and Klippenstein, S. J., "Comprehensive $\mathrm{O}_{2} / \mathrm{H}_{2}$ Kinetic Model for High-Pressure Combustion," International Journal of Chemical Kinetics, Vol. 44, No. 7, 2012, pp. $444-474$. doi: $10.1002 /$ kin.v44.7

[19] Kee, R. J., Grcar, J. F., Smooke, M. D., and Miller, J. A., "A Fortran Program for Modeling Steady Laminar One-Dimensional Premixed Flames," Sandia National Labs. Rept. SAND89-8240, Albuquerque, NM, 1985.

[20] Burcat, A., "Third Millennium Ideal Gas and Condensed Phase Thermochemical Database for Combustion," Technion-Israel Inst. of Technology, Aerospace Engineering Rept. 867, Haifa, Israel, Jan. 2001.

[21] White, F. M., "Preliminary Concepts," Viscous Fluid Flow, 2nd ed., McGraw-Hill, New York, 1991, pp. 29, 32.

[22] Trautz, M., and Melster, A., "Die Reibung, Wärmeleitung und Diffusion in Gasmischungen," Annalen der Physik, Vol. 399, No. 4, 1930, pp. 409-426. doi:10.1002/(ISSN)1521-3889

[23] Lindsay, A. L., and Bromley, L. A., "Thermal Conductivity of Gas Mixtures," Industrial and Engineering Chemistry, Vol. 42, No. 8, 1950, pp. $1508-1511$. doi:10.1021/ie50488a017

[24] Warnatz, J., Mass, U., and Dibble, R. W., "Transport Phenomena," Combustion, 2nd ed., Springer, New York, 1996, pp. 54, 56.

[25] Zhukov, V. P., "Verification, Validation, and Testing of Kinetic Mechanisms of Hydrogen Combustion in Fluid-Dynamic Computations," ISRN Mechanical Engineering, Vol. 2012, 2012, Paper 475607. doi: $10.5402 / 2012 / 475607$

J. Oefelein Associate Editor 